\title{
Implementation of Invigilation System using Face Detection and Face Recognition Techniques. A Case Study
}

\author{
Kathi Mohan Goud* and Shaik Jakeer Hussain \\ Department of ECE, VFSTR, Vadlamudi, Guntur,A.P,India-522213
}

Received 26 December 2020; Accepted 26 June 2021

\begin{abstract}
In recent years, face detection and face recognition techniques are improved sufficiently to make use in real-time applications and in crucial computer vision tasks. In this paper, the approaches that can use in real-time are discussed and implemented in a real-time application - Invigilation system. The web framework Django is used in designing the invigilation system and the database used is Mysql for storing the student and faculty data. The drawback of the traditional system is manual and failed to notify the wrong student attending the exam. This paper presents a method for automatic and optimised allotment into rooms and invigilators having face recognition for the student's correct prediction. Face detection is crucial for face recognition. To get the quick processing, an efficient, speed and accurate method was found by processing different images with various faces and found that the HOG method is best suited for processing a vast number of images. The face recognition model used in this paper has an accuracy of $99.38 \%$, which is sufficient for proper identification. The cameras placed in the rooms can take the pictures and send them to the Django server. The server processes the images, the face detector extracts the faces from the image and the face recognizer compares them with the faces of the allotted from the database. The whole system can identify the wrong person and able to find the attendees list. In future, it can develop in identifying the malpractices by implementing the tracking algorithms.
\end{abstract}

Keywords: Computer vision, Face detection, Face Recognition, HoG, Haar Cascade, CNN, 128D embeddings extraction, Student allotment.

\section{Introduction}

Examinations are the traditional way to scrutinize the students. The three essential steps in Examination conduction are Allotment, Monitoring and Evaluation. The manual procedure of allotment takes more time, requires human resources, and the chance of faults is more. The automatic allotment[9] is very advantageous, which involves the proper distribution of students considering his/her year of study, branch etc., and easily feasible with present technology. The purpose of monitoring is authentication and supervision. The manual monitoring involves an invigilator for checking the student's hall ticket, id card etc. and paying continuous attentiveness for avoiding the malpractice. Computer vision developed a lot in recent days. The monitoring phase can be automated using computer vision techniques. Face detection and Face recognition can aid in successful authentication. But supervision involves many detection techniques like have a camera and active internet connection during the exam. It is not possible to observe the student's surroundings which makes it easier to commit malpractice. Hence, it is difficult to avoid cheating in webcam-based invigilation systems[8]. The last step of Examinations is Evaluation. Automatic evaluation is $100 \%$ success for the objective, but no best approach is found for subjective still. Some of the methods which use feature extraction[24] are achieved some success. The design of the software tool[13] for only a

*E-mail address: kathi.mohangoud@gmail.com ISSN: 1791-2377 @ 2021 School of Science, IHU. All rights reserved. doi: $10.25103 /$ jestr.145.13 detecting the movement, whispering, face pose, abnormal behaviour, which are still under development. A candidate's movement can be detected by evaluating the difference between two successive frames and drawing contours. Open pose model can aid in detecting the pose. Abnormal behaviour can be estimated through emotion analysis. But the reliability of all these methods is not sufficient to use in the real-time. So avoiding an invigilator for monitoring is entirely not possible. But Remote proctoring can eliminate the invigilator's physical presence in the examination hall. Also, make it possible to conduct the examination for remote students which is unavoidable during situations like COVID. Online examinations are experienced by many threats. There were many possible ways of effectively confronting the attacks[15]. The usage of cryptographic technology[14] can aid in the security of online examinations. Invigilator, free fraudster detection system, is possible by the use of webcams [17]. The disadvantage of this system is every student must specific subject can aid in successful assessment. Overall the challenge of descriptive evaluation is still ON. Also eschewing invigilator during invigilations completely is not a good idea. But detecting the erroneous students is entirely possible with webcams through face recognition. The first step in face recognition is face detection. Many techniques were developed for efficient face detection. In knowledge-based face detection, certain rules like detection of eyes, nose at proper distances are framed and checked to identify a face. The practical implementation of such practices is difficult, and it gives many false positives. Compared to the knowledge-based method, the feature-based process is more successful, which is implemented by 
extracting the features of a face. Haar cascade method is an example of a feature-based method. Template matching method is the easiest way to implement. In this method, the frontal Face is predefined with a function but provides inadequate face detection results. Compared to all, the appearance-based method is the best-performed face detection method. It also used in face recognition. In this method, instead of defining a face template by experts, many training images are used for defining a face model and is achieved by applying Statistical analysis and Machine learning techniques. The popular methods for face detection are Haar Cascades[22], HoG and CNN[23].

\subsection{Face detection using Haar cascades method}

The face is said to be detected if the features of the face are found to be noticed. Haar cascade method perceives these features by Haar features or Haar wavelets. The face may be of any size and at any location in the image. Hence, the image is converted into different sized subwindows and all the Haar features are checked to match in the subwindow. One of the sub-window of Fig1 is shown in Fig 2. Since there were $1600+$ Haar features, it takes more time for the detection process to complete. Hence, the most relevant features are checked first. To do this, all the Haar features are divided into different stages based on their priority. If it passes all the stages, then the face is found in that specific sub-window, illustrated in Fig 4. A single face would be detected by many subwindows as shown in Fig3. All the concentric subwindow detections are neglected and treated to be one face. All such subwindows are called neighbours. There will be false positives if only one such neighbour is considered. For example, in Fig 5, three false-positive faces are detected. For least minimum neighbours, the false positives are more, and for huge minimum neighbours, the face detection is not accurate. Hence the minimum neighbours selection is optimum to detect the exact faces present, which is illustrated in Figs 5,6 and 7.

\subsection{Face detection using $\mathrm{CNN}$}

Dlib provides face detection using $\mathrm{CNN}$, which is a deep neural network trained with millions of images. The stored, trained .dat model is loaded and used for face detection. This model can find even the odd faces that cannot detect with HoG and Haar cascades. But it is very slow with CPUs. Fig 8 illustrates the implementation of CNN based face detector.

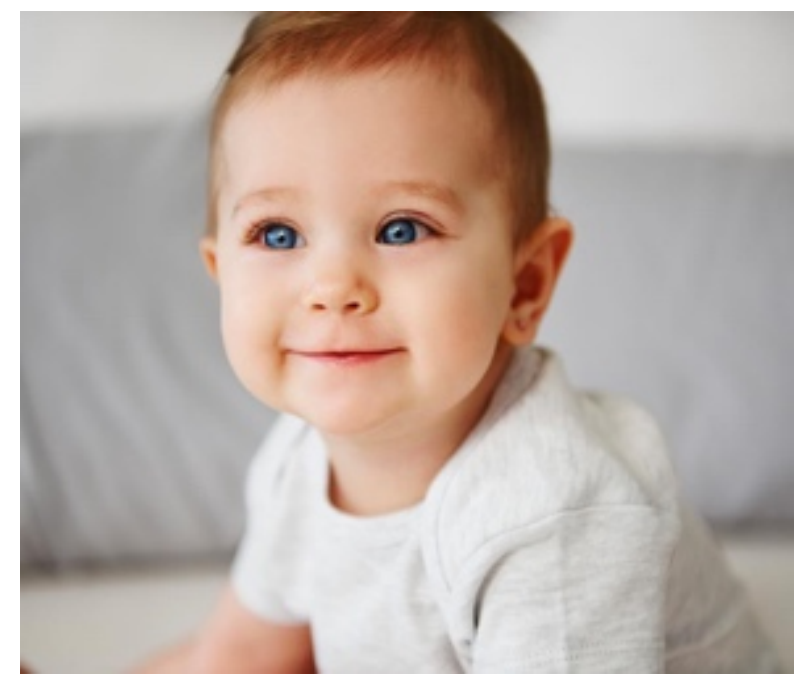

Fig. 1. Full image

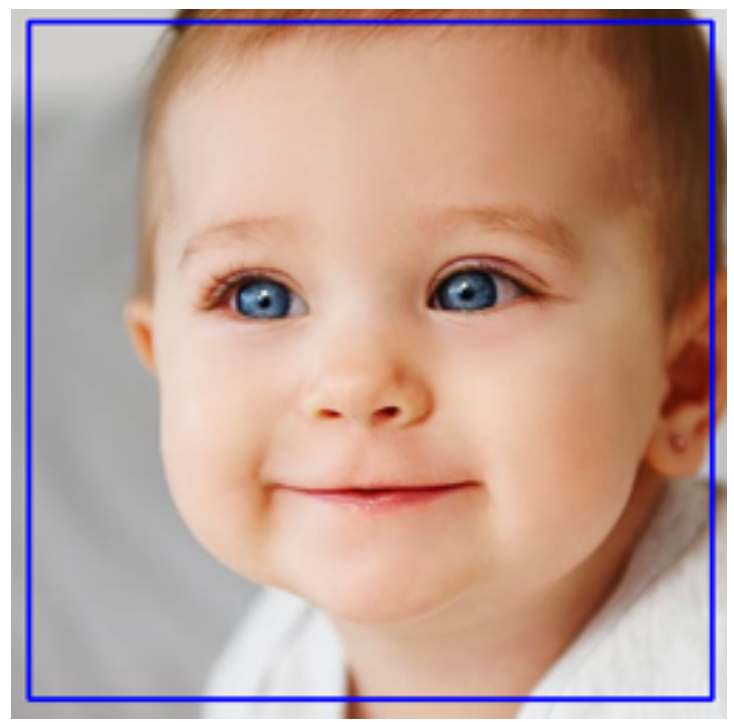

Fig. 2. Sub window

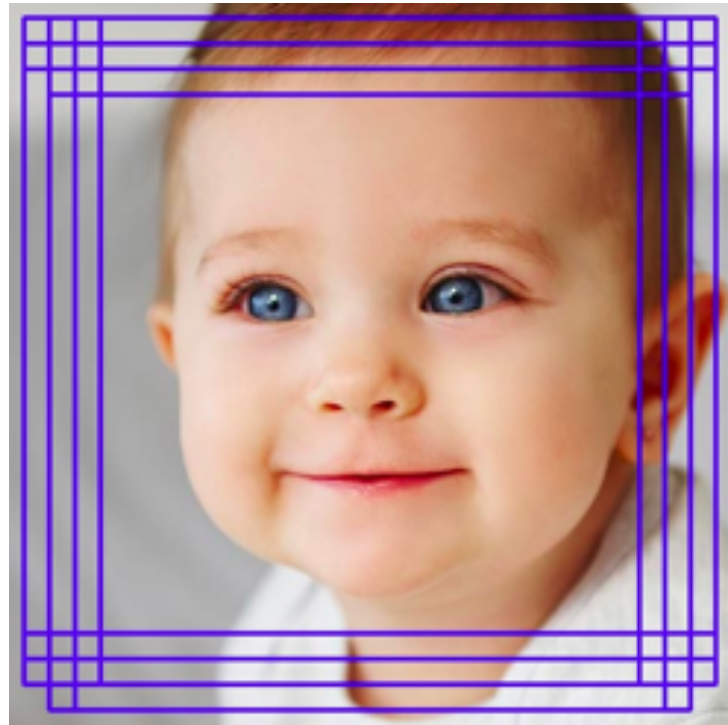

Fig. 3. All the sub windows which detects the face

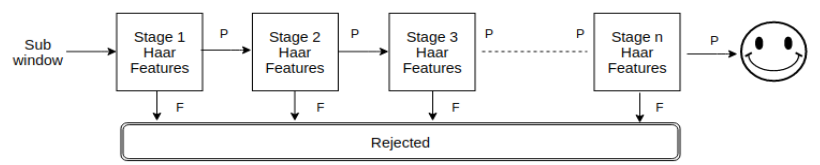

Fig. 4. Illustration of stages of Haar features for detecting the face in a subwindow

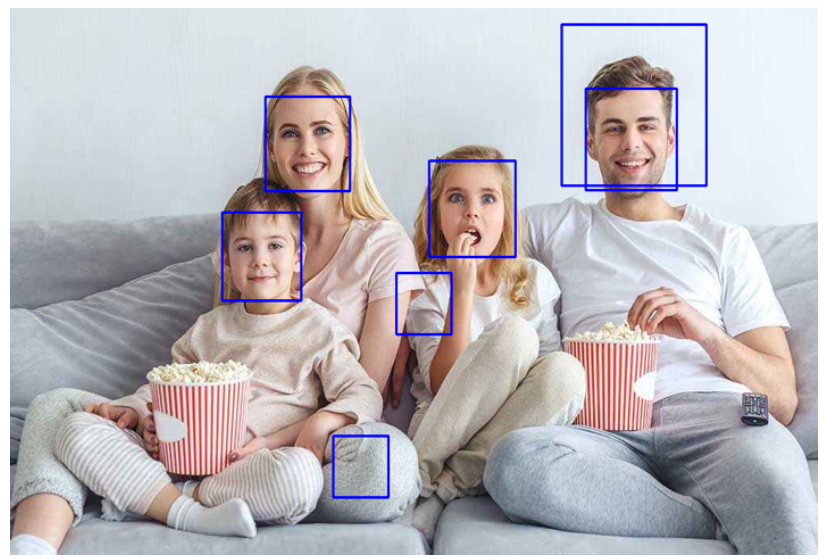

Fig .5. Minimum Neighbours -1 


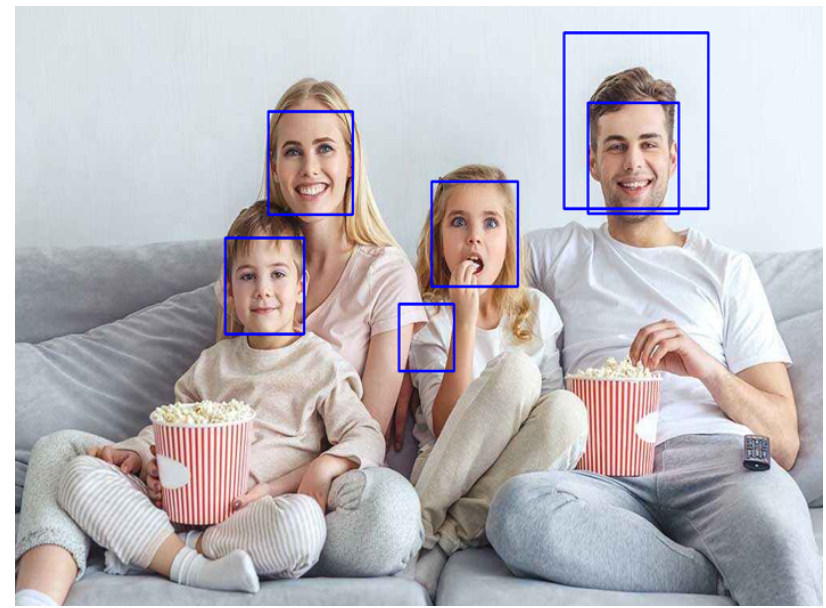

Fig. 6. Minimum Neighbours - 2

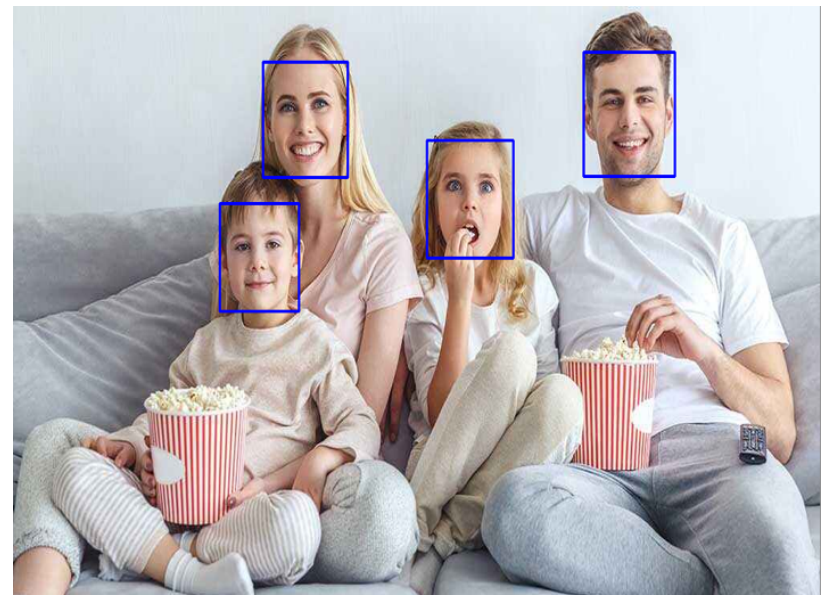

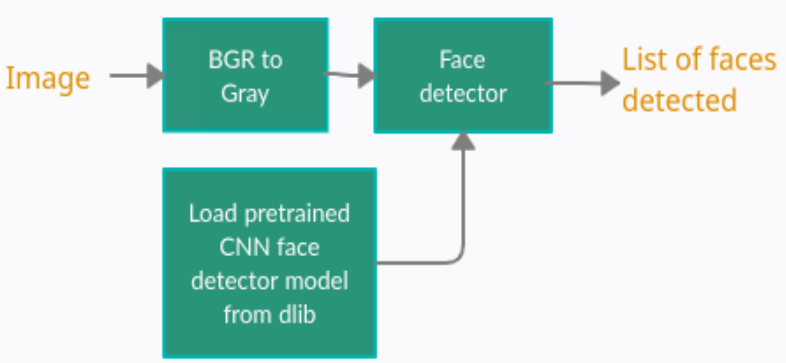

Fig. 8. Implementation of $\mathrm{CNN}$ face detector.

\subsection{Face detection using Histogram of Oriented} Gradients (HOG)

Gamma correction can enhance the image quality[25]. For gamma correction, the pixel intensities are scaled from $[0$ $255]$ to [0 1].

The scaled output image is defined as (scaled input image $)^{1 / g}$ and is rescaled in [ $\left[\begin{array}{ll}0 & 255\end{array}\right]$ to obtain the output image.

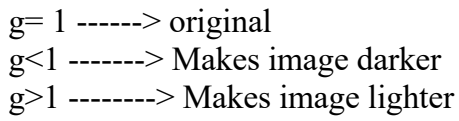

The image at different values of $\mathrm{g}$ is illustrated in Fig 9. It is found that $g=1.5$ is good for most of the images. Fig10 illustrates the improvement in images when $\mathrm{g}=1.5$.

Before proceeding with the HoG feature representation, the image is resized to $64 \times 128$ because the image is divided into $8 \times 8$ and $16 \times 16$ patches for extracting the features.

Fig. 7. Minimum Neighbours - 3

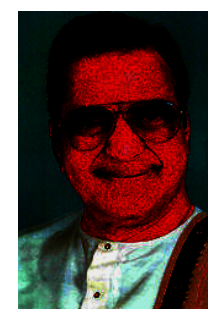

$\mathrm{g}=0.1$

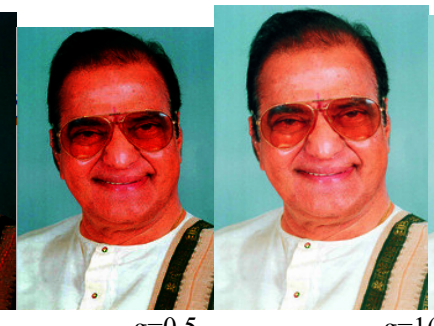

$\mathrm{g}=0.5$ $\mathrm{g}=1$ (original) $\mathrm{g}=1.5 \quad \mathrm{~g}=2$

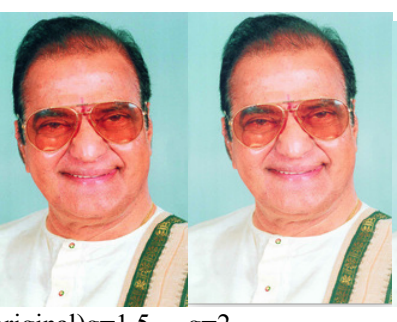

$g=2$

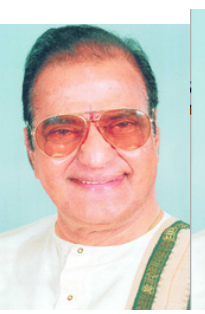

$\mathrm{g}=2.5$

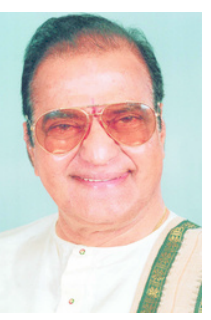

$\mathrm{g}=3$

Fig. 9. Image enhancement at different gamma corrections

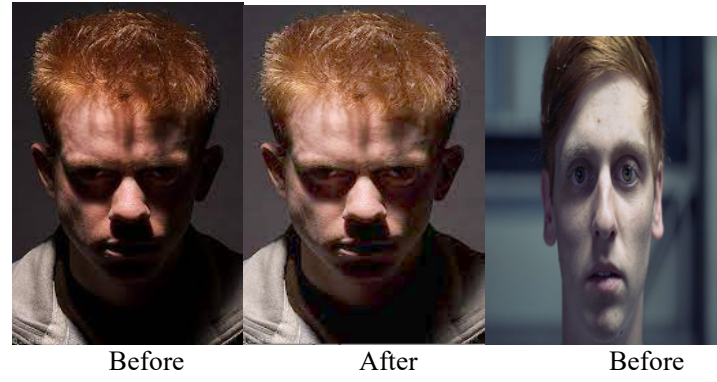

Fig. 10. Improvement in various images due to gamma correction $(g=1.5)$

HOG is a very useful image descriptor. HOG represents as a single vector for the entire image. It's computed by sliding window detector over an image and generate a HOG descriptor for each position in the image, and each position is combined with the single vector later on. The pyramiding technique is beneficial for generating this. $\mathrm{HOG} s$ are traditionally best used with SVM. By comparing the HOG

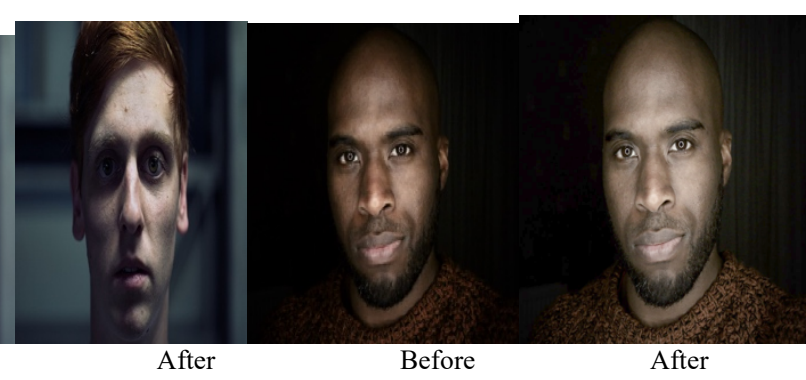

patterns of the image with the HOG patterns from the trained data set, the faces in the image can be detected. The procedure of detecting the face is explained by using an algorithm ALG1.

\section{ALG 1}

1: Convert RGB to gray

2: Calculate the horizontal( $\mathrm{HG})$ and vertical(VG) gradients. 
3: Calculate the gradient magnitude $=\sqrt{V G^{2}+H G^{2}}$ and gradient angle $=\tan ^{-1}(\mathrm{VG} / \mathrm{HG})$

4: Disintegrate the image into $16 \times 16$ pixels and replace the $16 \times 16$ pixels block with a gradient of resultant of gradient of each pixel of all the $16 \times 16$ pixels.

5: Plot the hog features of original image as in Fig 11, and compare it with the known HoG pattern (Fig 12) which is produced from the training images.

The face detector that uses HOG method detects the faces in the given image and extracts the images for identification as illustrated with the help of Fig 13 and 14.

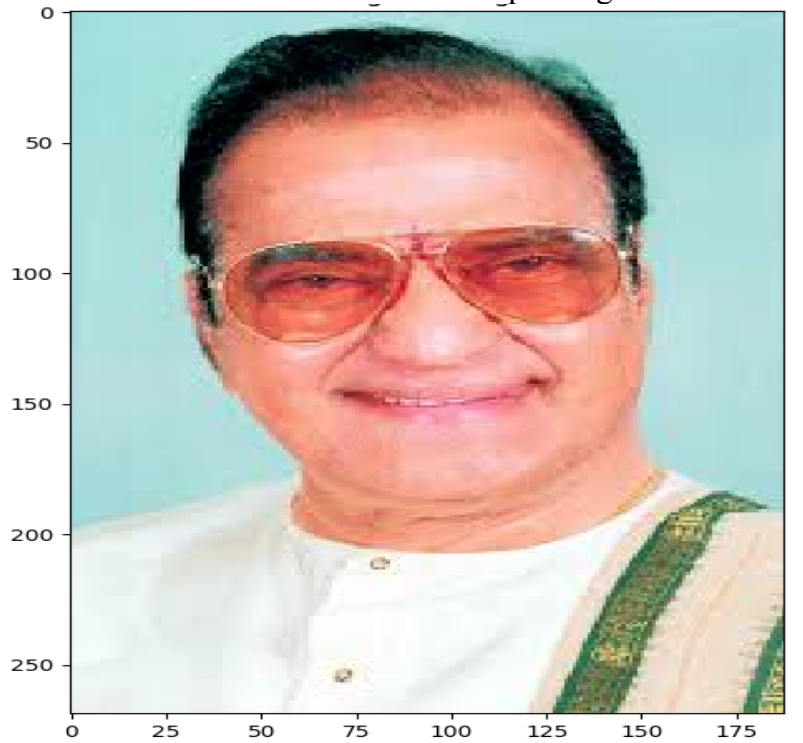

Fig. 11. Original Image and its HOG feature representation
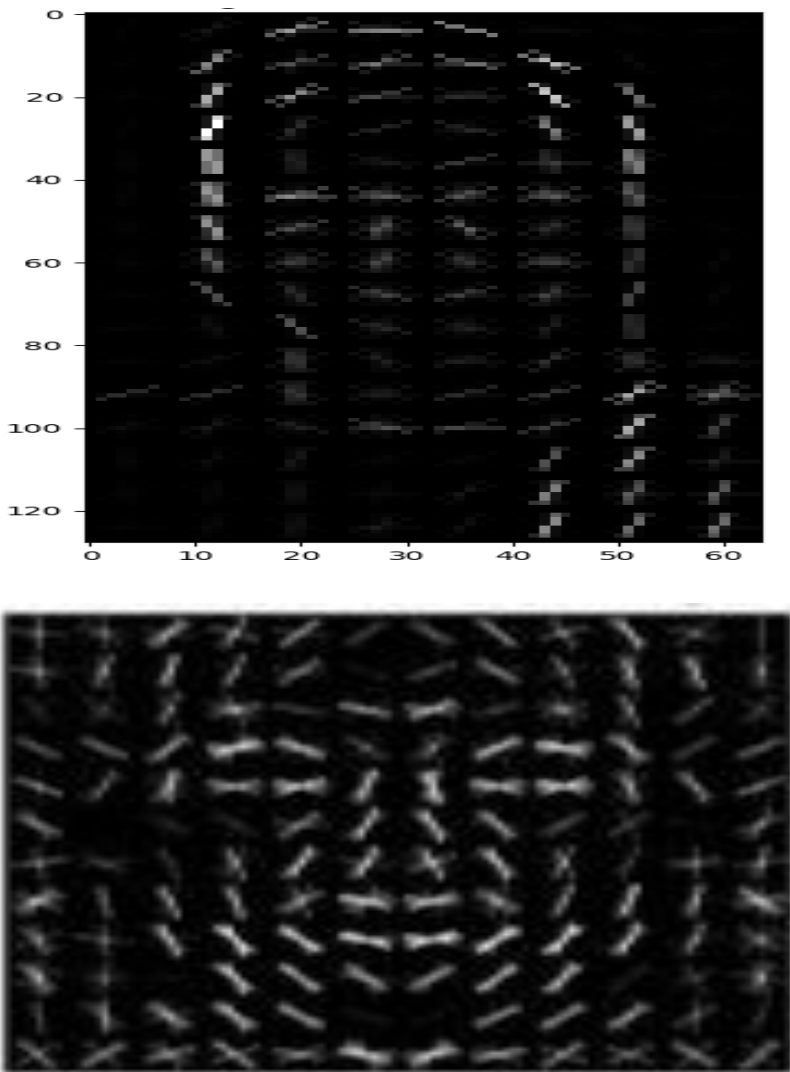

Fig. 12. HOG features extracted from training images

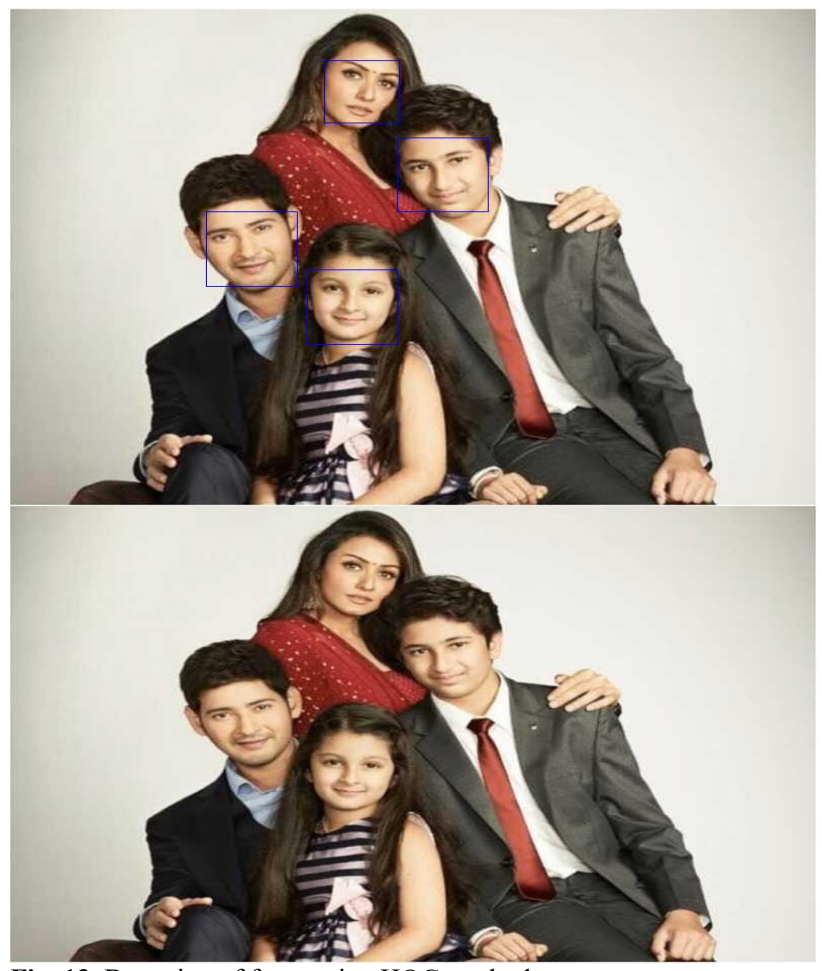

Fig. 13. Detection of faces using HOG method.

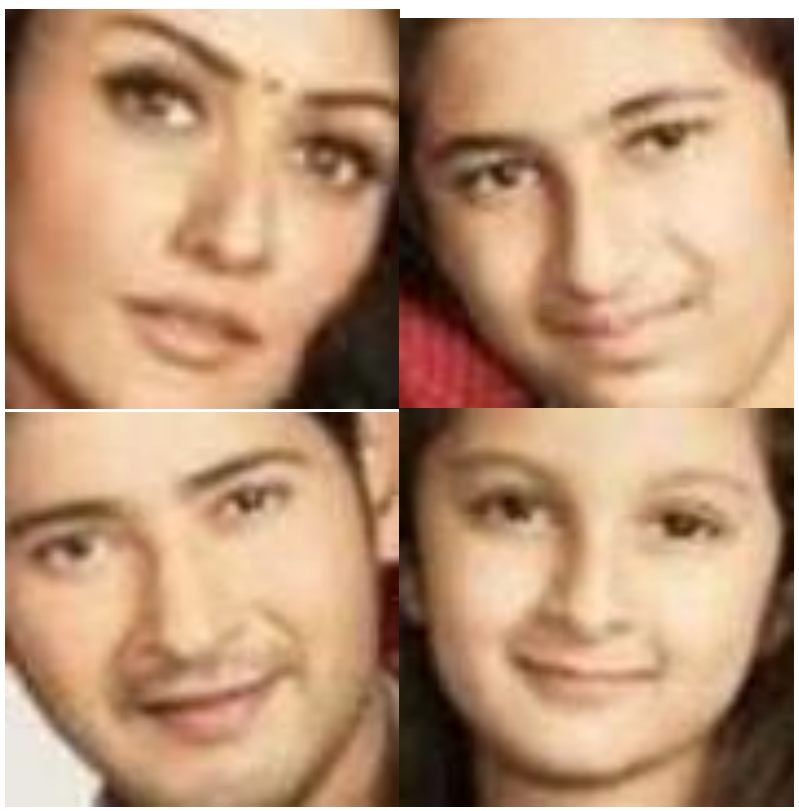

Fig. 14. Extracted faces of Fig 13 using HOG method.

\section{Speed and Пerformance Comparison}

The popular methods for face detection are Haar Cascades[22], HoG and CNN[23]. The speed of detecting the face is crucial as the images to be processed increases. To predict the faster method, Five categories of images having one, two, three, four and five faces are taken and calculated the speed of detection using these three methods which are illustrated in Figures 15-24. In Graphs CNN, HoG and Haar cascade methods' speeds are represented with yellow, Green, and Blue. From Figs 15 to 19, it is found that CNN is much slower compared to all. Since the time taken for CNN to detect face is very high compared to other methods, Figs 15 to 19 were not properly shown the speed differences of HOG 
and Haar Cascade methods. Hence, they were compared separately in Figs 20 to 24. The speeds are taken without considering the classifier loading time. The classifier loading time for Haar, $\mathrm{HoG}$ and $\mathrm{CNN}$ is $0.016,0.317$ and 0.075 seconds, respectively. For the given images the speeds without considering classifier loading times are between 0.007 and 0.19 for Haar, 0.069 and 3.14 for $\mathrm{HoG}$ and between 1.46 and 90.94 for CNN. Since classifier will be loaded only once, it only affects the first image's speed. Hence this effect is neglected. The fastest among all is Haar, followed by HoG and CNN. Haar and HoG methods don't use much CPU resources, but CNN affects speed of other running processes on the same CPU.

Even though Haar cascades method is faster, it requires the scale factor and minimum neighbours as inputs. For example, for Fig 24, the correct prediction of faces, the scale factor and minimum neighbours required is 1.2 and 3 (Table 1) but is not valid for all the cases. For example, for Fig 25, it is 1.11 and 3 . So the chance of false detection is more in this method. Hence, interns of speed even though Haar is faster, HoG is best to use.

With the increase in faces in the image, the face detection time increases, which can't be shown with these graphs because of only 1 to 5 faces of image difference. To notice the difference, Consider Figs 25 and 26, the faces detected in Fig26 is 215 at 4.73 seconds and Fig 25 is 7 faces at 0.5 seconds by HoG method.

In order to predict the best performer in terms of accuracy and precision, 1015 images are considered. The TP, FN and accuracy values of these approaches are illustrated in Table2.It is found that CNN is best in terms of accuracy and sensitivity but consideration of speed makes it worse and It is found that HoG is best suited for real time applications when all the performance measures are take into the account.

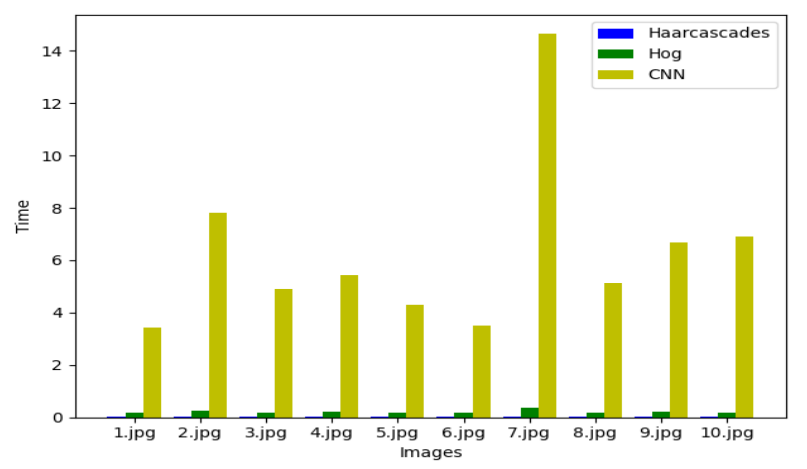

Fig. 15. Single face image comparison of all the methods

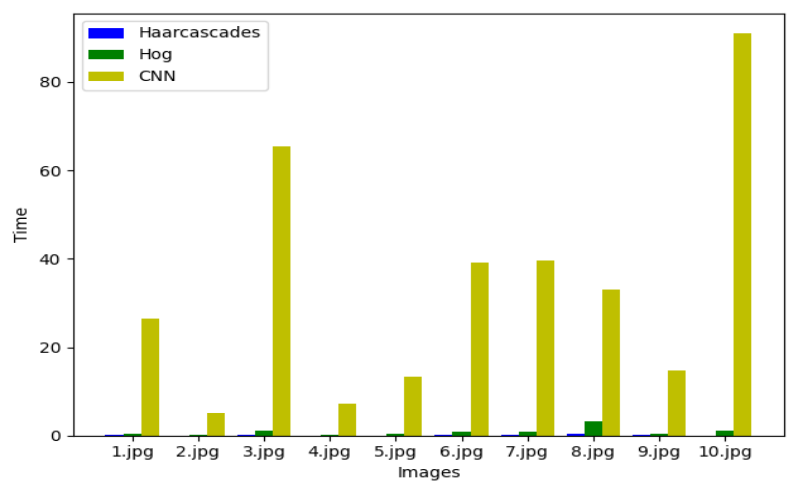

Fig. 16. Two face image comparison of all the methods

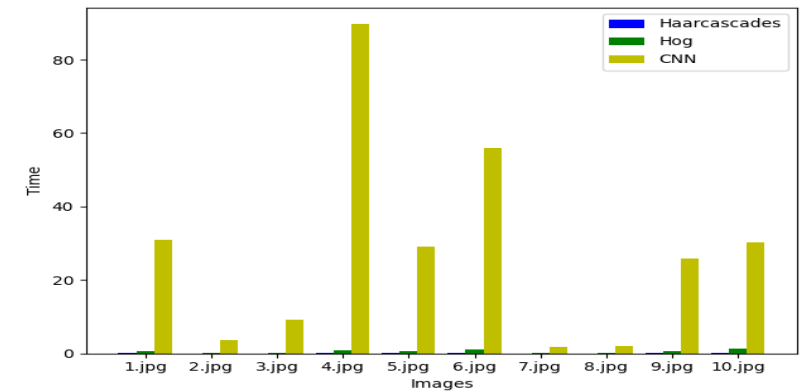

Fig. 17. Three Face image comparison of all the methods

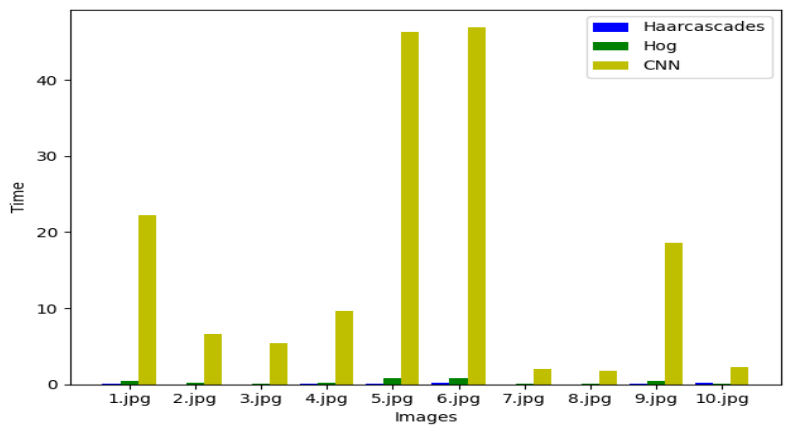

Fig. 18. Four Face image comparison of all the methods

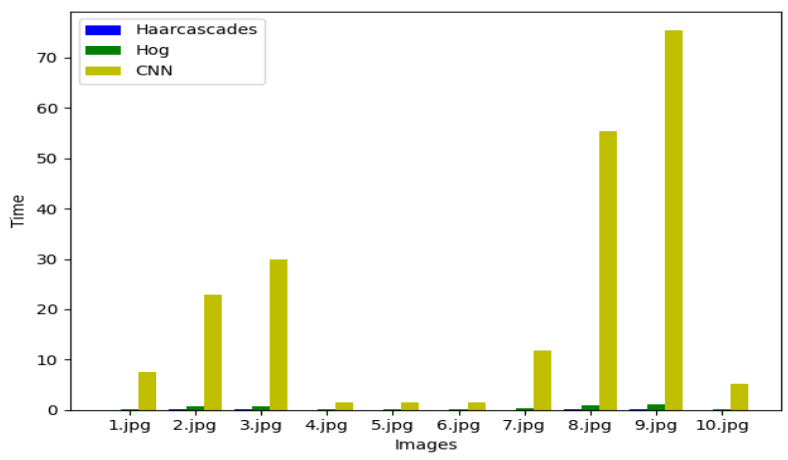

Fig. 19. Five Face image comparison of all the methods

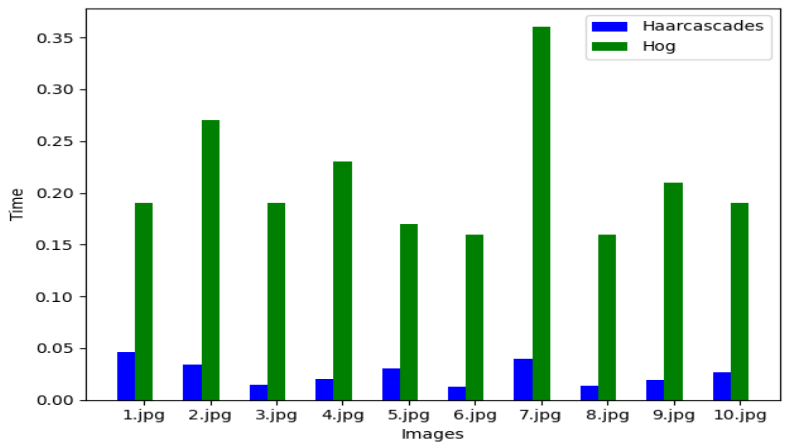

Fig. 20. Single face image comparison of Haar and Hog methods

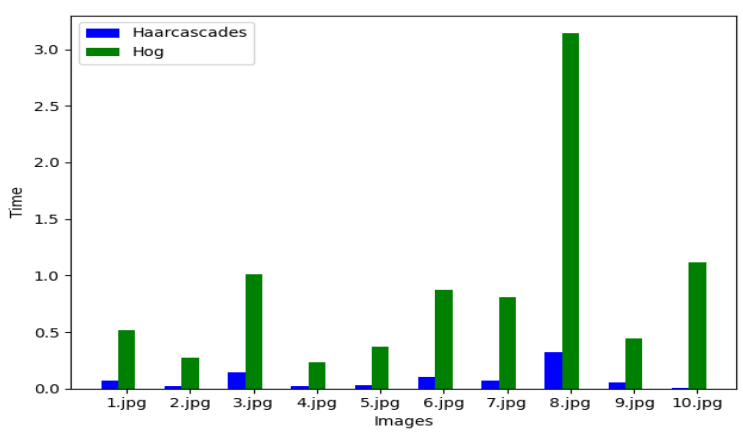

Fig. 21. Two face image comparison of Haar and Hog 


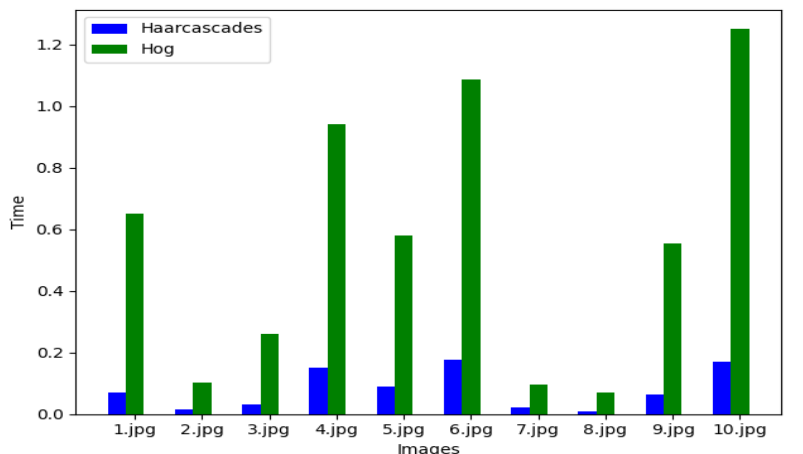

Fig. 22. Three face image comparison of Haar methods and Hog methods

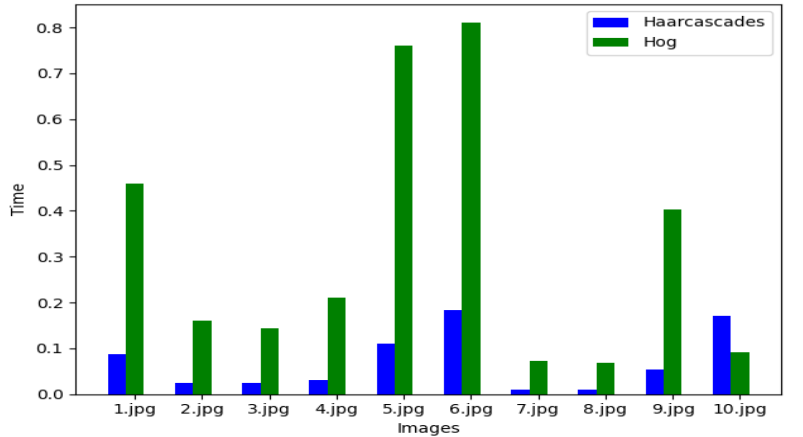

Fig. 23. Four Face image comparison of Haar and Hog

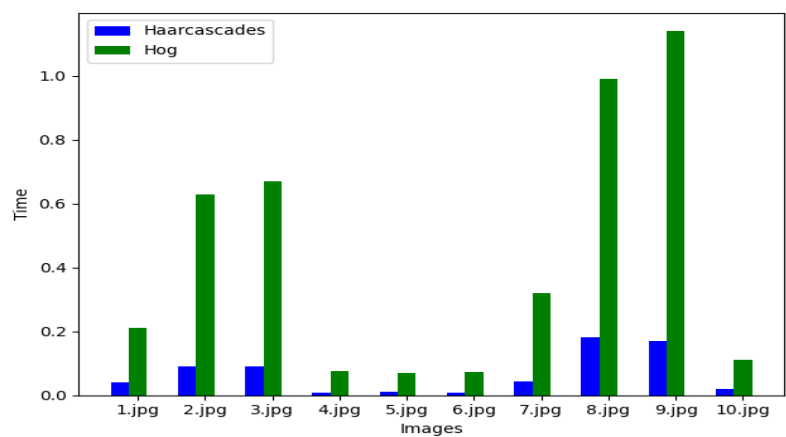

Fig. 24 .Five Face image comparison of Haar methods and Hog methods

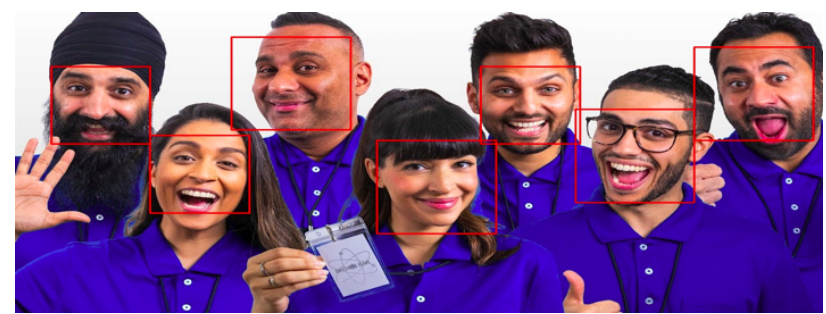

Fig. 25. Face detection for less images

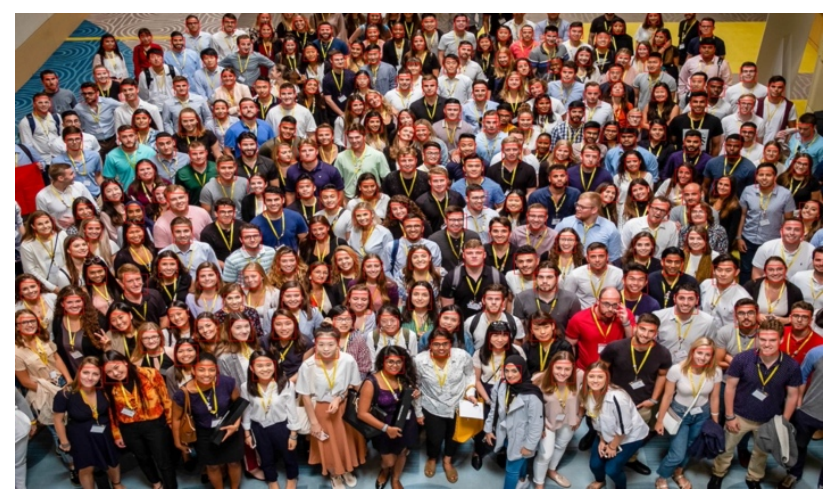

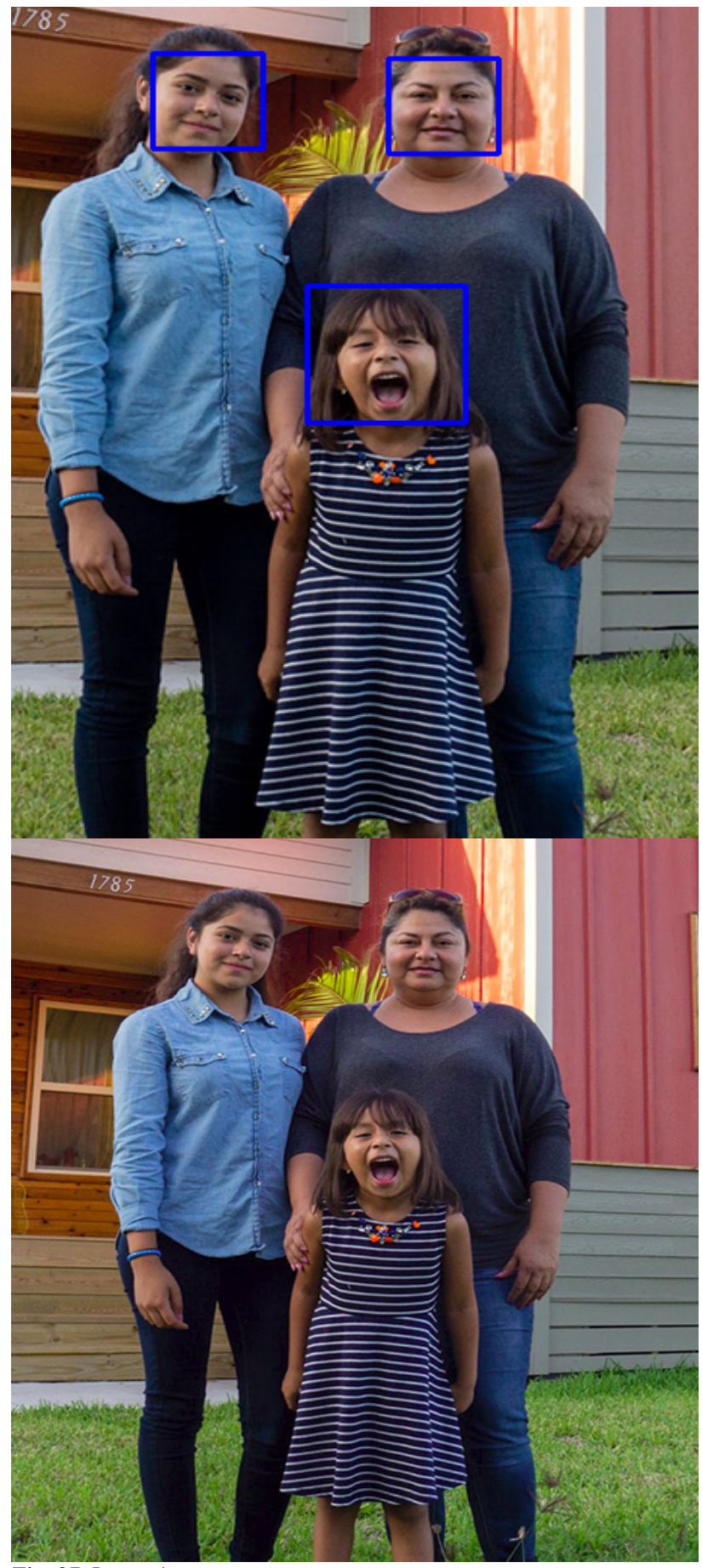

Fig. 27. Image 1

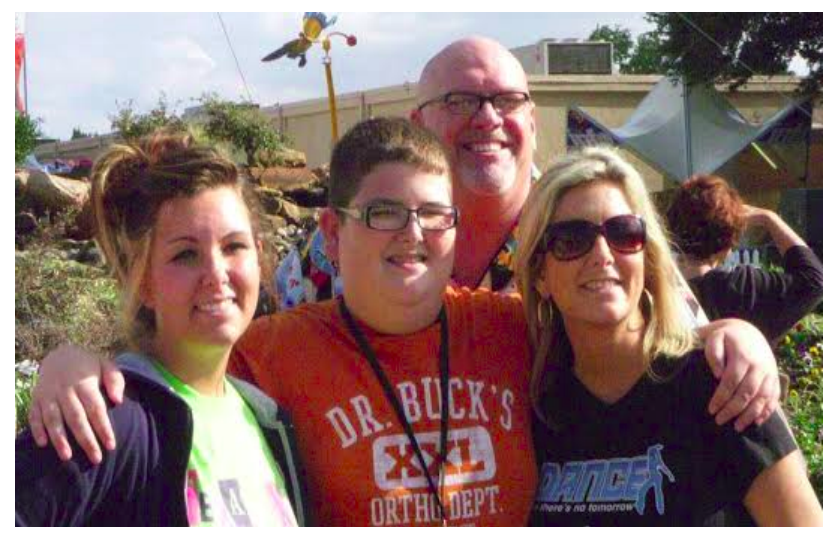

Fig. 26. Face detection for huge number of images 


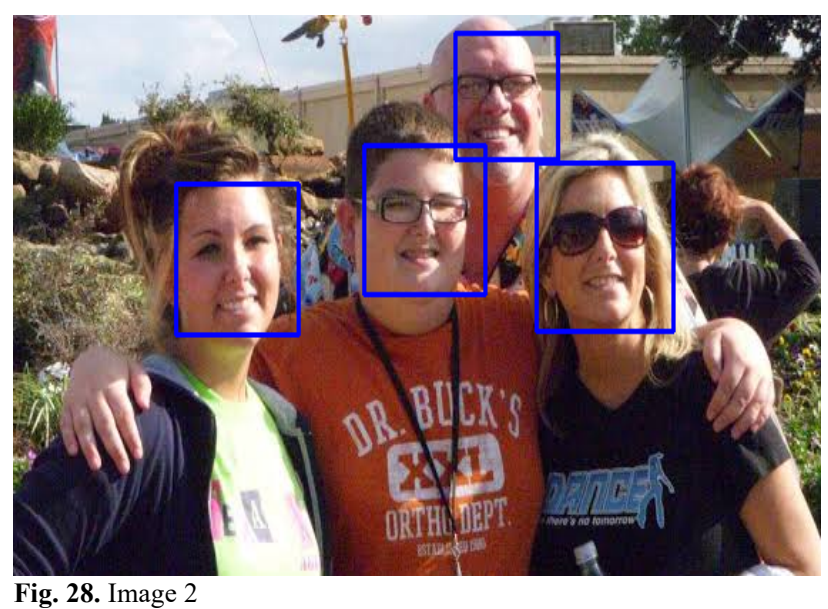

Table 1. Scale factor and Minimum Neighbours selection

\begin{tabular}{l|c|l|l}
\hline Scale Factor & $\begin{array}{l}\text { Minimum } \\
\text { Neighbours }\end{array}$ & $\begin{array}{l}\text { Faces } \\
\text { detected for } \\
\text { Fig 27 }\end{array}$ & $\begin{array}{l}\text { Faces } \\
\text { detected for } \\
\text { Fig 28 }\end{array}$ \\
\hline 1.1 & 1 & 7 & 5 \\
1.1 & 2 & 6 & 3 \\
1.1 & 3 & 4 & 3 \\
1.11 & 3 & 4 & 4 \\
1.2 & 3 & 3 & 3 \\
\hline
\end{tabular}

Table 2. TP, FN, Accuracy and Sensitivity values of face detection techniques.

\begin{tabular}{l|l|l|l|l|l}
\hline Method & TP & FN & Accuracy & Sensitivity & Sensitivity \\
\hline HoG & 994 & 21 & 97.93 & 97.93 & 97.93 \\
CNN & 1013 & 2 & 99.8 & 99.8 & 99.8 \\
$\begin{array}{l}\text { Haar } \\
\text { Cascade }\end{array}$ & 874 & 141 & 86.1 & 86.1 & 86.1 \\
\hline
\end{tabular}

\section{Rotation of face}

In order to identify the face recognizer more accurately. The extracted faces are rotated so that the eyes are in a straight line with mouth centred. The rotation of face is applied by identifying the 68 landmark points in the face, which is illustrated in Fig 29. The points 37 to 40 represents a right eye, 34 to 48 represents a left eye, and 49 to 61 represents the mouth. The centre of the image coordinates is half of extreme $\mathrm{x}$ and $\mathrm{y}$ coordinates of the image. The mouth is centred by shifting the average of 53 and 57 coordinates to centre coordinates and the eyes are straightened by making the y coordinates of $37,40,43$ and 46 as same.

\section{Face Recognition}

There were different methods for face recognition[10]. [3] proposes a new descriptor for face recognition, [19] introduces IPCA-ICA algorithm for face recognition. Infrared based face recognition is best suited compared to the visible spectrum[4]. [16] proposes a method of face recognition based on Infrared. It isn't easy to recognize the face if the whole frontal face is not observed. By projecting $3 \mathrm{D}$ shape in a $2 \mathrm{D}$ plane, the face contour can be detected, which can help recognise the faces at different poses[6]. Even though the whole frontal face is not seen, the face can be recognized through the gait[21]. But expensive in 3D discourages in embedded applications. It is possible to detect the face at different poses without using 3D. The developments are made in the face technology under various illumination conditions[1] for indoor[2] too. [5] proposes a method for recognising the face at different ages. [20] proposes a method for face, with the addition of the tracking algorithm[11] can use in humanoid robots. One of the accurate methods for recognizing the face is by finding the $128 \mathrm{D}$ embeddings in a face using deep neural network and calculating the euclidean distance between them which is illustrated in Fig 30, and the same is used in this paper. presentation attack detection. After recognizing the

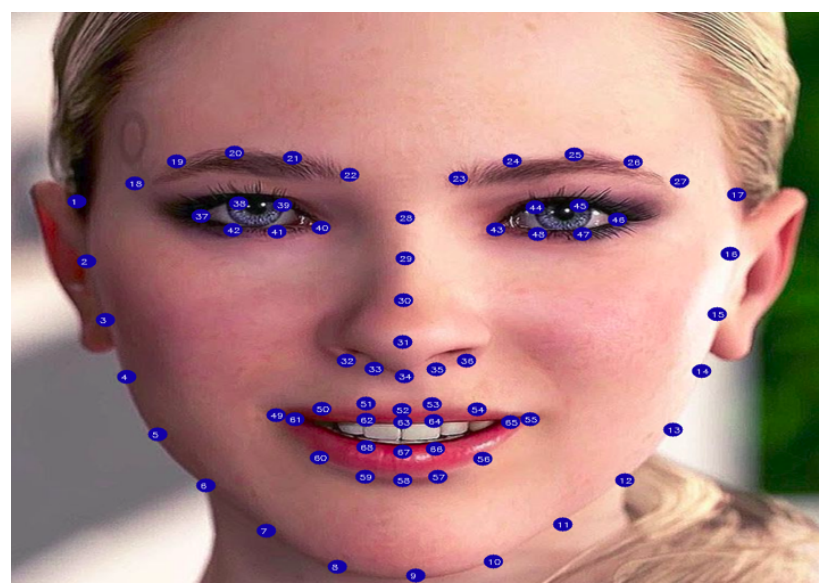

Fig 29. Representation of 68 landmarks on face.

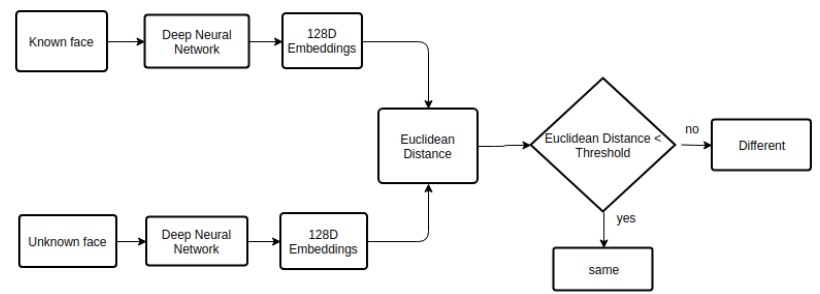

Fig. 30. Face Recognizer.

The deep neural network outputs a feature vector instead of the single label from the inputted image. The feature vector is $128 \mathrm{D}$ embeddings which are illustrated in Fig 31. The neural network used is based on RESNET-34. Approximately 3,000,000 images are used for training the network. The training image sets consists of three image per set. Two are of the same face and the third is of a different face. The network is trained such that the embeddings are nearer for identical faces and far for the negative face. $99.38 \%$ is the accuracy achieved on the standard Label faces in the wild (LFW) benchmark. In LFW, a total of 1680 people having distinct images are present which are used for testing purposes. The Euclidean distance between the known and unknown images $=\sqrt{\sum_{i=1}^{128}\left(a_{i}-b_{i}\right)^{2}}$ and is compared with the given threshold and if the Euclidean distance is found to be less than the threshold. The face is said to be matched. 


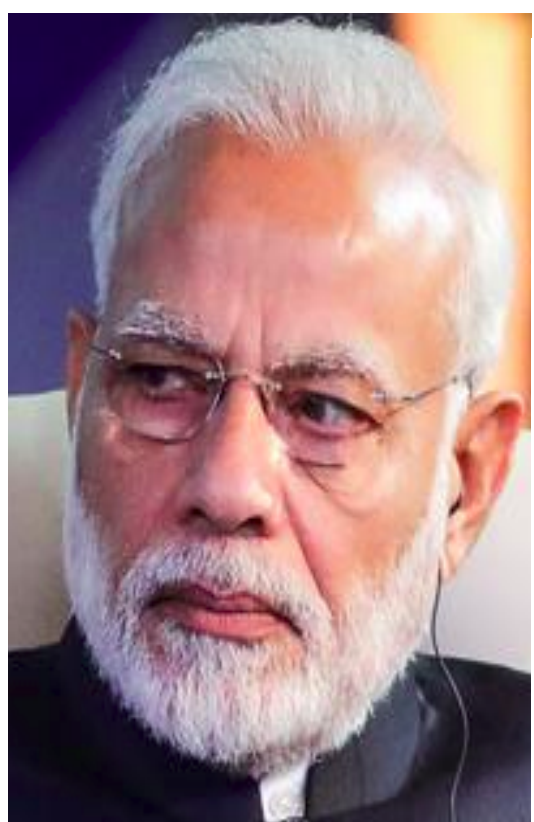

Fig. 31. 128D embeddings(RHS) of the image(LHS)

$\begin{array}{cccccc}{[-0.10021268} & 0.08632077 & 0.07127149 & -0.09811363 & 0.00628576 & -0.08542342 \\ 0.03628761 & -0.07716832 & 0.16281596 & -0.00510453 & 0.1988795 & 0.05139653 \\ -0.25384539 & -0.10366542 & -0.03337254 & 0.05847298 & -0.1706275 & -0.14096098 \\ -0.08608174 & -0.11783254 & 0.00446755 & 0.02538201 & 0.11356518 & 0.01784196 \\ -0.11056112 & -0.32919392 & -0.08706772 & -0.20556653 & 0.01134552 & -0.07470456 \\ 0.03990082 & 0.00086641 & -0.20252401 & -0.0846408 & -0.00736513 & 0.00880007 \\ -0.05507532 & -0.04181438 & 0.18054858 & 0.029357 & -0.14144906 & 0.03854407 \\ 0.04984567 & 0.25425923 & 0.22538815 & 0.05365215 & 0.02251036 & -0.09543332 \\ 0.01163939 & -0.21982391 & 0.1278915 & 0.14543675 & 0.02804084 & 0.07484955 \\ 0.04529105 & -0.12017966 & 0.04025063 & 0.08790777 & -0.24348299 & 0.08417476 \\ 0.08178685 & -0.1338968 & -0.07751781 & 0.03014585 & 0.1634526 & 0.14059269 \\ -0.10835868 & -0.1124603 & 0.11371122 & -0.18852189 & -0.06464984 & 0.06026264 \\ -0.10198241 & -0.12505533 & -0.23122405 & 0.08557017 & 0.39747676 & 0.14037168 \\ -0.09188332 & 0.03678616 & -0.19502766 & 0.00132257 & 0.04930104 & 0.03236819 \\ -0.10928129 & -0.06474142 & -0.0995875 & 0.03959223 & 0.10273 & 0.02752331 \\ -0.10778718 & 0.13972944 & -0.0531284 & 0.01162232 & 0.05514103 & 0.07902336 \\ -0.14237715 & 0.01432091 & -0.12805486 & -0.06020878 & 0.03291136 & -0.08462083 \\ -0.02099959 & 0.05407608 & -0.16573566 & 0.09975535 & 0.01744767 & -0.09727282 \\ 0.01435585 & -0.01782359 & -0.0168467 & -0.02614969 & 0.10436933 & -0.23849337 \\ 0.31795567 & 0.20942375 & 0.0571523 & 0.18326725 & 0.07307366 & 0.0233093 \\ -0.03736403 & -0.05812167 & -0.13435642 & -0.06232781 & 0.02844457 & 0.08012772 \\ 0.14963138 & 0.01809811] & & & & \end{array}$

$\left[\begin{array}{llllll}-0.10021268 & 0.08632077 & 0.07127149 & -0.09811363 & 0.00628576 & -0.08542342\end{array}\right.$

$\begin{array}{llllll}0.08608174 & -0.11783254 & 0.00446755 & 0.02538201 & 0.11356518 & 0.01784196\end{array}$

$\begin{array}{lllllll}0.11056112 & -0.32919392 & -0.08706772 & -0.20556653 & 0.01134552 & -0.07470456\end{array}$

$\begin{array}{lllllll}0.03990082 & 0.00086641 & -0.20252401 & -0.0846408 & -0.00736513 & 0.00880007\end{array}$

$\begin{array}{llllll}0.01163939 & -0.21982391 & 0.1278915 & 0.14543675 & 0.02804084 & 0.07484955\end{array}$

$\begin{array}{lllllll}0.04529105 & -0.12017966 & 0.04025063 & 0.08790777 & -0.24348299 & 0.08417476\end{array}$

$\begin{array}{llllll} & \end{array}$

$\begin{array}{lllllll}0.10198241 & -0.12505533 & -0.23122405 & 0.08557017 & 0.39747676 & 0.14037168\end{array}$

$\begin{array}{llllll}0.09188332 & 0.03678616 & -0.19502766 & 0.00132257 & 0.04930104 & 0.03236819\end{array}$

$\begin{array}{llllll}0.10928129 & -0.06474142 & -0.09995875 & 0.03959223 & 0.10273 & 0.02752331\end{array}$

$\begin{array}{llllll}0.10778718 & 0.13972944 & -0.0531284 & 0.01162232 & 0.05514103 & 0.07902336\end{array}$

$\begin{array}{lllllll}-0.14237715 & 0.01432091 & -0.12805486 & -0.06020878 & 0.03291136 & -0.08462083\end{array}$

$\begin{array}{llllll}0.02099959 & 0.05407608 & -0.16573566 & 0.09975535 & 0.01744767 & -0.09727282\end{array}$

$0.01435585-0.01782359-0.0168467 \quad-0.02614969-0.10436933-0.23849337$

$\begin{array}{llllll}0.0 .03736403 & -0.05812167 & -0.13435642 & -0.06232781 & 0.02844457 & 0.08012772\end{array}$ $0.14963138 \quad 0.01809811]$
The performance measures that are used in [26] are used in estimating the performance of the face recognition technique. The confusion matrix is illustrated in Table 3 and the performance parameters used are:

Accuracy $=(\mathrm{TP}+\mathrm{TN}) /$ Total

Sensitivity or Recall or True positive rate $=\mathrm{TP} /$ actual correctperson $=\mathrm{TP} /(\mathrm{FN}+\mathrm{TP})$

specificity or selectivity or True negative rate $=\mathrm{TN} /$ actual wrong person $=\mathrm{TN} /(\mathrm{TN}+\mathrm{FP})$

Precision $=\mathrm{TP} /$ predicted correct person $=\mathrm{TP} /(\mathrm{FP}+\mathrm{TP})$

$\mathrm{f}$-score $=2 \mathrm{x}($ precisionxsensitivity $) /($ precision + sensitivi ty $)$

Total five celebrities(Emma Watson, Bill Gates, Obama, Shakira and Tom cruise) each of 100 images is used in analysing the performance. TP and TN values of each subject at different thresholds is illustrated in Table 4. The suffix letter (E-Emma Watson. B-Billgates, O-Obama, SShakira, T-TomCruise) represents the subject name. The values of FP and FN are Total-TP and Total-TN respectively.
The performance measures of each subject are illustrated with the help of Table 5,6,7,8 and 9. It is found that the $\mathrm{f}$ score and maximum accuracy achieved is at threshold 0.7 in the range of 97 to 99.5 . The sensitivity increases and specificity decreases with an increase in the threshold. But at 0.7 , the measure of positives identified correctly(sensitivity) and negatives identified correctly (specificity) is between 96 and 100 which is the best performance. Hence the threshold of 0.7 is used for face recognition.

Table 3. Confusion matrix of face recognition technique

\begin{tabular}{c|c|c|c}
\hline \multicolumn{2}{c}{} & \multicolumn{2}{c}{ Predicted } \\
\cline { 2 - 4 } \multicolumn{2}{c}{ Cofusion Matrix } & $\begin{array}{c}\text { Wrong } \\
\text { person }\end{array}$ & $\begin{array}{c}\text { Correct } \\
\text { person }\end{array}$ \\
\hline \multirow{4}{*}{ Actual } & $\begin{array}{c}\text { Wrong } \\
\text { person }\end{array}$ & $\mathrm{TN}$ & $\mathrm{FP}$ \\
\cline { 2 - 4 } & $\begin{array}{c}\text { Correct } \\
\text { person }\end{array}$ & $\mathrm{FN}$ & $\mathrm{TP}$ \\
\hline
\end{tabular}

TP-True Negative, TP-True Positive, FP-False Positive, FN-False Negative

Table 4. TP and TN at different thresholds.

\begin{tabular}{c|c|c|c|c|c|c|c|c|c|c|c}
\hline Threshold & $\mathbf{0}$ & $\mathbf{0 . 1}$ & $\mathbf{0 . 2}$ & $\mathbf{0 . 3}$ & $\mathbf{0 . 4}$ & $\mathbf{0 . 5}$ & $\mathbf{0 . 6}$ & $\mathbf{0 . 7}$ & $\mathbf{0 . 8}$ & $\mathbf{0 . 9}$ & $\mathbf{1}$ \\
\hline TPE & 0 & 0 & 0 & 0 & 8 & 56 & 91 & 99 & 100 & 100 & 100 \\
TNE & 100 & 100 & 100 & 100 & 100 & 100 & 100 & 96 & 85 & 37 & 3 \\
TPB & 0 & 1 & 6 & 6 & 11 & 65 & 96 & 100 & 100 & 100 & 100 \\
TNB & 100 & 100 & 100 & 100 & 100 & 100 & 100 & 97 & 81 & 53 & 16 \\
TPO & 1 & 1 & 1 & 5 & 39 & 79 & 97 & 99 & 100 & 100 & 100 \\
TNO & 100 & 100 & 100 & 100 & 100 & 100 & 100 & 100 & 86 & 46 & 6 \\
TPS & 0 & 0 & 0 & 0 & 26 & 78 & 97 & 100 & 100 & 100 & 100 \\
TNS & 100 & 100 & 100 & 100 & 100 & 99 & 99 & 96 & 80 & 43 & 5 \\
TPT & 0 & 0 & 0 & 1 & 10 & 53 & 95 & 100 & 100 & 100 & 100 \\
TNT & 100 & 100 & 100 & 100 & 100 & 100 & 100 & 99 & 75 & 46 & 15 \\
\hline
\end{tabular}


Table 5. Emma Watson

\begin{tabular}{l|l|l|l|l|l|l|l|l|l|l|l}
\hline Threshold & $\mathbf{0}$ & $\mathbf{0 . 1}$ & $\mathbf{0 . 2}$ & $\mathbf{0 . 3}$ & $\mathbf{0 . 4}$ & $\mathbf{0 . 5}$ & $\mathbf{0 . 6}$ & $\mathbf{0 . 7}$ & $\mathbf{0 . 8}$ & $\mathbf{0 . 9}$ & $\mathbf{1}$ \\
\hline Accuracy & 50 & 50 & 50 & 50 & 54 & 78 & 95.5 & 97.5 & 92.5 & 68.5 & 51.5 \\
Sensitivity & 0 & 0 & 0 & 0 & 8 & 56 & 91 & 99 & 100 & 100 & 100 \\
Specificity & 100 & 100 & 100 & 100 & 100 & 100 & 100 & 96 & 85 & 37 & 3 \\
Precision & $\mathrm{x}$ & $\mathrm{x}$ & $\mathrm{x}$ & $\mathrm{x}$ & 100 & 100 & 100 & 96 & 86 & 61 & 50 \\
F Score & $\mathrm{x}$ & $\mathrm{x}$ & $\mathrm{x}$ & $\mathrm{x}$ & 14 & 71 & 95 & 97 & 93 & 76 & 67 \\
\hline
\end{tabular}

Table 6. Bill Gates

\begin{tabular}{l|l|l|l|l|l|l|l|l|l|l|l}
\hline Threshold & $\mathbf{0}$ & $\mathbf{0 . 1}$ & $\mathbf{0 . 2}$ & $\mathbf{0 . 3}$ & $\mathbf{0 . 4}$ & $\mathbf{0 . 5}$ & $\mathbf{0 . 6}$ & $\mathbf{0 . 7}$ & $\mathbf{0 . 8}$ & $\mathbf{0 . 9}$ & $\mathbf{1}$ \\
\hline Accuracy & 50 & 50.5 & 53 & 53 & 55.5 & 82.5 & 98 & 98.5 & 90.5 & 76.5 & 58 \\
Sensitivity & 0 & 1 & 6 & 6 & 11 & 35 & 96 & 100 & 100 & 100 & 100 \\
Specificity & 100 & 100 & 100 & 100 & 100 & 100 & 100 & 97 & 81 & 53 & 16 \\
Precision & $\mathrm{x}$ & 100 & 100 & 100 & 100 & 100 & 100 & 97 & 84 & 68 & 54 \\
F Score & $\mathrm{x}$ & 1 & 11 & 11 & 19 & 78 & 97 & 98 & 91 & 80 & 70 \\
\hline
\end{tabular}

Table 7. Obama

\begin{tabular}{l|l|l|l|l|l|l|l|l|l|l|l}
\hline Threshold & $\mathbf{0}$ & $\mathbf{0 . 1}$ & $\mathbf{0 . 2}$ & $\mathbf{0 . 3}$ & $\mathbf{0 . 4}$ & $\mathbf{0 . 5}$ & $\mathbf{0 . 6}$ & $\mathbf{0 . 7}$ & $\mathbf{0 . 8}$ & $\mathbf{0 . 9}$ & $\mathbf{1}$ \\
\hline Accuracy & 50.5 & 50.5 & 50.5 & 52.5 & 69.5 & 89.5 & 98.5 & 99.5 & 93 & 73 & 53 \\
Sensitivity & 1 & 1 & 1 & 5 & 39 & 79 & 97 & 99 & 100 & 100 & 100 \\
Specificity & 100 & 100 & 100 & 100 & 100 & 100 & 100 & 100 & 86 & 46 & 6 \\
Precision & 100 & 100 & 100 & 100 & 100 & 100 & 100 & 100 & 87 & 64 & 51 \\
F Score & 1 & 1 & 1 & 9 & 56 & 89 & 98 & 99 & 93 & 78 & 68 \\
\hline
\end{tabular}

Table 8. Shakira

\begin{tabular}{l|l|l|l|l|l|l|l|l|l|l|l}
\hline Threshold & $\mathbf{0}$ & $\mathbf{0 . 1}$ & $\mathbf{0 . 2}$ & $\mathbf{0 . 3}$ & $\mathbf{0 . 4}$ & $\mathbf{0 . 5}$ & $\mathbf{0 . 6}$ & $\mathbf{0 . 7}$ & $\mathbf{0 . 8}$ & $\mathbf{0 . 9}$ & $\mathbf{1}$ \\
\hline Accuracy & 50 & 50 & 50 & 50 & 63 & 89 & 98 & 98 & 98 & 71.5 & 52.5 \\
Sensitivity & 0 & 0 & 0 & 0 & 26 & 78 & 97 & 100 & 100 & 100 & 100 \\
Specificity & 100 & 100 & 100 & 100 & 100 & 99 & 99 & 96 & 80 & 43 & 5 \\
Precision & $\mathrm{x}$ & $\mathrm{x}$ & $\mathrm{x}$ & $\mathrm{x}$ & 100 & 98 & 98 & 96 & 83 & 63 & 51 \\
F Score & $\mathrm{x}$ & $\mathrm{x}$ & $\mathrm{x}$ & $\mathrm{x}$ & 41 & 87 & 97 & 98 & 90 & 77 & 67 \\
\hline
\end{tabular}

Table 9. Tom Cruise

\begin{tabular}{l|l|l|l|l|l|l|l|l|l|l|l}
\hline Threshold & $\mathbf{0}$ & $\mathbf{0 . 1}$ & $\mathbf{0 . 2}$ & $\mathbf{0 . 3}$ & $\mathbf{0 . 4}$ & $\mathbf{0 . 5}$ & $\mathbf{0 . 6}$ & $\mathbf{0 . 7}$ & $\mathbf{0 . 8}$ & $\mathbf{0 . 9}$ & $\mathbf{1}$ \\
\hline Accuracy & 50 & 50 & 50 & 50.5 & 55 & 76.5 & 97.5 & 99.5 & 87.5 & 73 & 57.5 \\
Sensitivity & 0 & 0 & 0 & 1 & 10 & 53 & 95 & 100 & 100 & 100 & 100 \\
Specificity & 100 & 100 & 100 & 100 & 100 & 100 & 100 & 99 & 75 & 46 & 15 \\
Precision & $\mathrm{x}$ & $\mathrm{x}$ & $\mathrm{x}$ & 100 & 100 & 100 & 100 & 99 & 80 & 64 & 54 \\
F Score & $\mathrm{x}$ & $\mathrm{x}$ & $\mathrm{x}$ & 1 & 18 & 69 & 97 & 99 & 89 & 78 & 70 \\
\hline
\end{tabular}

\section{Procedure of Allotment}

Django is used for storing the students and faculty data and for the procedure of allotment. It is an open-source web application framework which can even run in single board computers for applications like bicycle care[12]. In [7], the database data is communicated with SQL. It is difficult for the programmer to learn one language for website maintenance and other for database related queries. Hence
Django is used, in which database queries can be managed with python instead of using SQL. Also, it is easy to change from one database to other without any code changes. Some of the databases that are supported by Django are sqlite, mysql, postgress etc.,. The Rooms data is stored in the database as rows and columns with the Room Name. Students data as student name, Roll number, branch, year, section and photo. Similarly, the faculty data is stored in the 
database with his name, designation and branch with an image.

Students are allotted into the exam halls following the algorithm ALG2. For efficient allocation, the sections having the highest students is allocated first. Care is taken to prevent students belong to the same section come back to back or side by side or diagonal.

\section{ALG2}

Step1: Total seats count $\mathrm{k}=\sum_{\mathrm{i}=1}{ }^{\mathrm{n}}\left(\mathrm{R}_{\mathrm{i}} \mathrm{X} \mathrm{C} \mathrm{C}_{\mathrm{i}}\right)$. Where, $\mathrm{n}$ is the total number of rooms, $R_{i}$ and $C_{i}$ are the number of rows and columns of the $\mathrm{i}^{\text {th }}$ room respectively. Check whether students count $>\mathrm{k}$. if true, print error message and exit, else proceed with step2.

Step2: s be the students data as the combination of year and branch in descending order of the combination count. Let the combination be c. s1,s2,s3 and $s 4 \in \mathrm{s}$ are the selectors. And each selector consists of one of c's list which is not allotted. An offset say o is maintained for $\mathrm{s}$ to find upto which combination, the allocation is completed.

Step3: Select the first room.

Step4: If the selected room's rows are the multiples of three then fill the rows and columns of room with students in selectors as

if $\mathrm{R}_{(\mathrm{i}-1)(\mathrm{jmax})}=\mathrm{s}_{\mathrm{p}}$ then

$R_{i j}=S_{p} \quad$ if $j=4 n$ where $n=1,2,3$, ---

$\mathrm{s}_{\mathrm{p}+1}($ for $\mathrm{p}+1<5)$ ors $\mathrm{p}-3 \quad$ if $\mathrm{j}=4 \mathrm{n}-3$

$s_{p+2}($ for $p+1<5)$ ors $s_{p-2} \quad$ if $j=4 n-2$

$s_{p+3}($ for $p+1<5)$ ors $s_{p-1} \quad$ if $j=4 n-1$

else if not the multiples of three, follow the below combination by taking three selectors

if $R_{(i-1)(j \max )}=s_{p}$ then

$R_{i j}=s_{p} \quad$ if $j=3 n$ where $n=1,2,3,---$

$\mathrm{s}_{\mathrm{p}+1}($ for $\mathrm{p}+1<5)$ ors $\mathrm{p}_{\mathrm{p}-2} \quad$ if $\mathrm{j}=3 \mathrm{n}-2$

$s_{p+2}($ for $p+1<5)$ ors $s_{p-1} \quad$ if $j=3 n-1$

where $\mathrm{p}$ is 1 or 2 or 3 or 4 , jmax is the maximum number of columns in the room.

The student data is removed from a selector once it was allotted. If a selector is empty, it is filled with c's list from s and $o$ is improved by 1 .

Step 5: If at least one student $\in \mathrm{s}$ is not allotted by the end of total seats filled in the room. Select the next Room and repeat step 4. Store all the room names which are used for
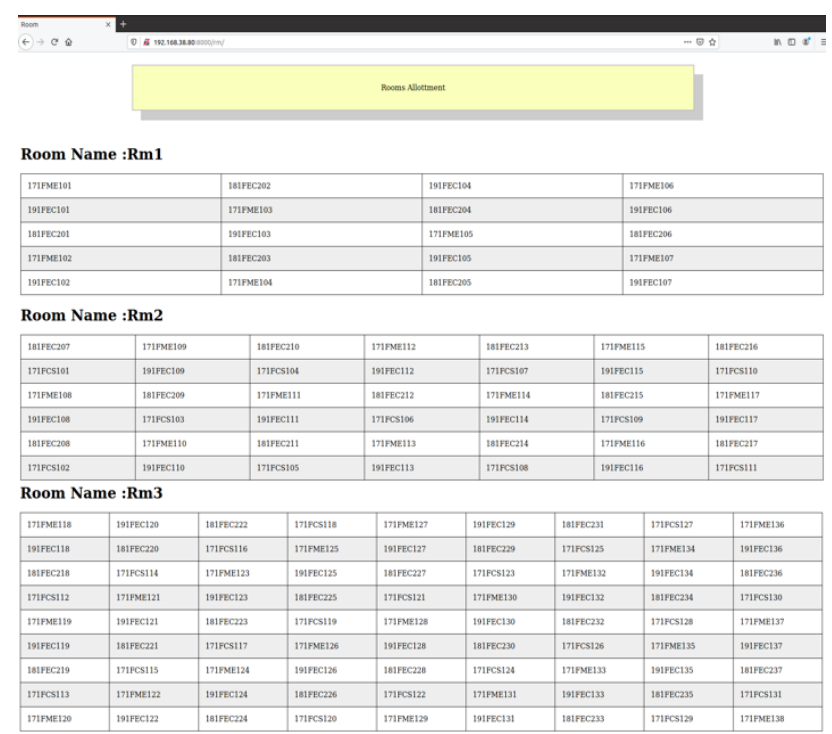

allotment in a list say $r$ and exit if all the students are allotted.

Similarly, invigilators are allotted for the students occupied rooms as per the algorithm ALG3.

\section{ALG 3}

Step 1: Total number of invigilators needed is $T_{i}=\frac{1}{30} \sum_{i=1}{ }^{n}$ $\left(\mathrm{R}_{\mathrm{i}} \mathrm{X} \mathrm{C}_{\mathrm{i}}\right)$. Check $\mathrm{T}_{\mathrm{i}}<\mathrm{T}$ where $\mathrm{T}$ is the total faculty. If true proceed with step 2 else exit with an error message.

Step 2 : Initialize an offset say fi to track the faculty and select the first room from $r$.

Step 3 : Number of faculty needed for a room $\mathrm{rf}=\frac{1}{30}(\mathrm{RxC})$. Allot the faculty of $\mathrm{T}$ from fi to fi+rf and update fi value with fi + rf .

Step 4 : Check whether all the rooms are allotted. If yes, exit. Else select next room and repeat from step 3.

\section{Results and discussion}

The students data that used is Mechanical the third year from Roll no. 171FME101 to 171FME167, EEE third year from Roll no. 171FME101 to 171FME159, CSE third year from Roll no. 171FCS101 to 171FCS160, CSE second year from Roll no. 181FCS201 to 181FCS253, ECE first year from Roll no. 191FEC101 to 191FEC162,ECE second year from Roll no. 181FEC201 to 181FEC260,ECE third year from Roll no. 171FEC301 to $171 \mathrm{FEC} 359$ and the rooms taken are RM1 of $5 \times 4$ where 5 is rows and 4 is columns whose total strength is $5 \times 4=20, \mathrm{RM} 2$ of $6 \times 7, \mathrm{RM} 3$ of $9 \times 9$, RM4 of $7 \times 7$, RM5 of $6 \times 4$, RM6 of 7x9, RM of $6 \times 4$, RM8 of 9x8, RM9 of $7 \times 9$. The Rooms allotment as per the specified algorithm, seating allotment and the invigilator allotment are presented in Fig 32,33 and 34 respectively. The camera placed inside each room takes pics continuously and send to the server. The server detects the faces using the face detector and separates all the faces separately. The last step is the identification, each face separated is compared with the each of roll number's photo that is allotted in that room. If matches then that person is marked as present and this process continues for the first hour of the examination and stores the final attendance after half an hour.
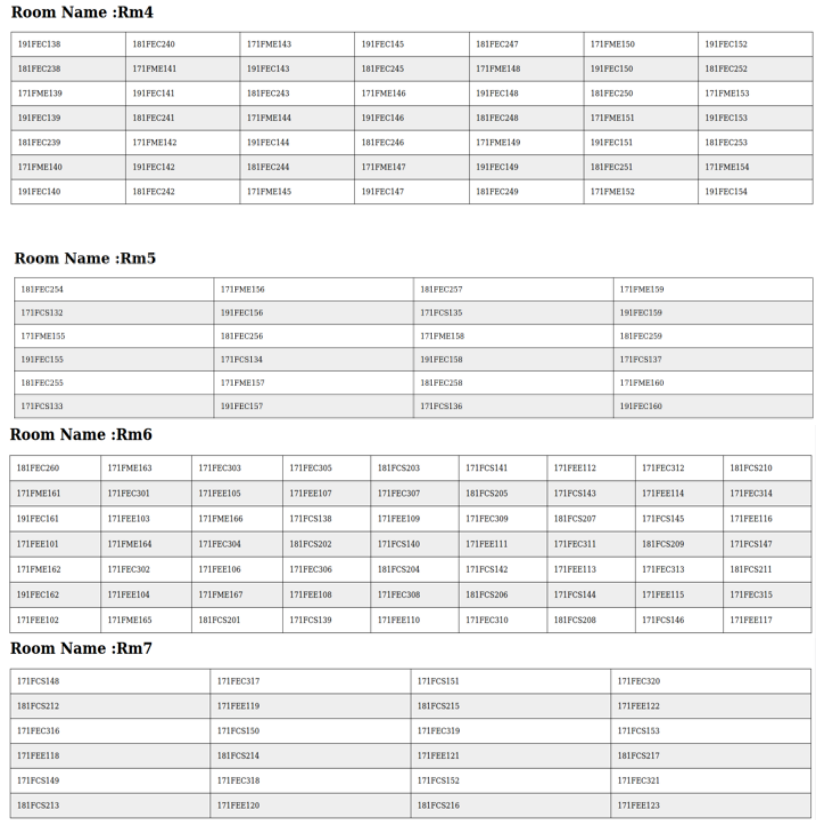


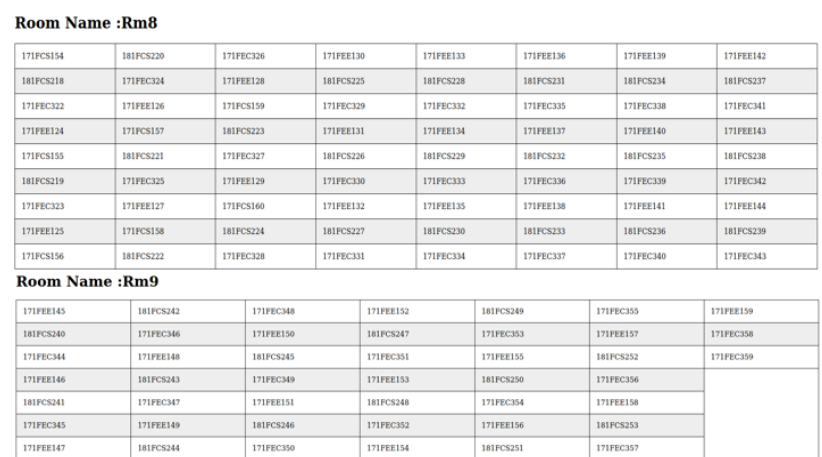

Fig 32. Rooms Allotment

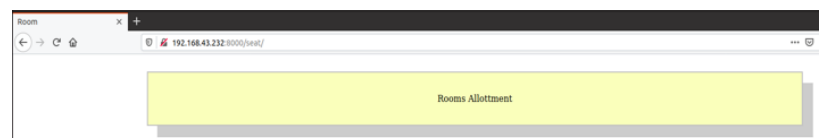

Room Name : Rm1 Room Name : Rm2

171FME101 - 171FME107

191 FEC101 - 191 FEC107

171FME 108 - 171FME 11

$191 \mathrm{FEC} 108$ - $191 \mathrm{FEC} 117$

$181 \mathrm{FEC} 207-181 \mathrm{FEC} 17$

171FCS101 - 171FCS111

\section{Room Name :Rm3}

171FME118 - 171FME138

\section{Room Name : Rm4}

$191 \mathrm{FEC} 118$ - $191 \mathrm{FEC} 137$

171FME139 - 171FME154

$191 \mathrm{FEC} 138$ - $191 \mathrm{FEC} 154$

$181 \mathrm{FEC} 218-181 \mathrm{FEC} 23$

$181 \mathrm{FEC} 238-181 \mathrm{FEC} 253$

\section{Room Name : Rm6}

\section{Room Name :Rm5}

E161 - 171FME167

191 FEC161 - 191FEC162

181 FEC260 - 181 FEC260

171 FEE 101 - 171 FEE117

$171 \mathrm{FEC} 301$ - $171 \mathrm{FEC} 315$

$181 \mathrm{FCS} 201$

$171 \mathrm{FCS} 138-171 \mathrm{FCS} 147$

171FCS132 - 171FCS137

\section{Room Name :Rm7}

171FEE118 - 171FEE123

171FEC316 - 171FEC321

Room Name : $\operatorname{Rm} 8$

181 FCS212 - 181FCS217

171FEE124 - 171 FEE 144

$171 \mathrm{FEC} 322$ - $171 \mathrm{FEC} 343$

181 FCS218 - 181FCS239

171FCS148 - 171FCS153

$171 \mathrm{FCS} 154-171 \mathrm{FCS} 160$

\section{Room Name :Rm9}

171FEE145 - 171FEE159

171 FEC 344 - 171FEC359

$181 \mathrm{FCS} 240$ - 181FCS253

Fig. 33. Seating plan

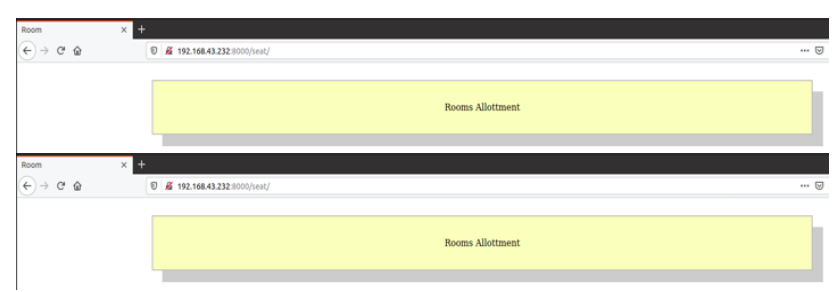

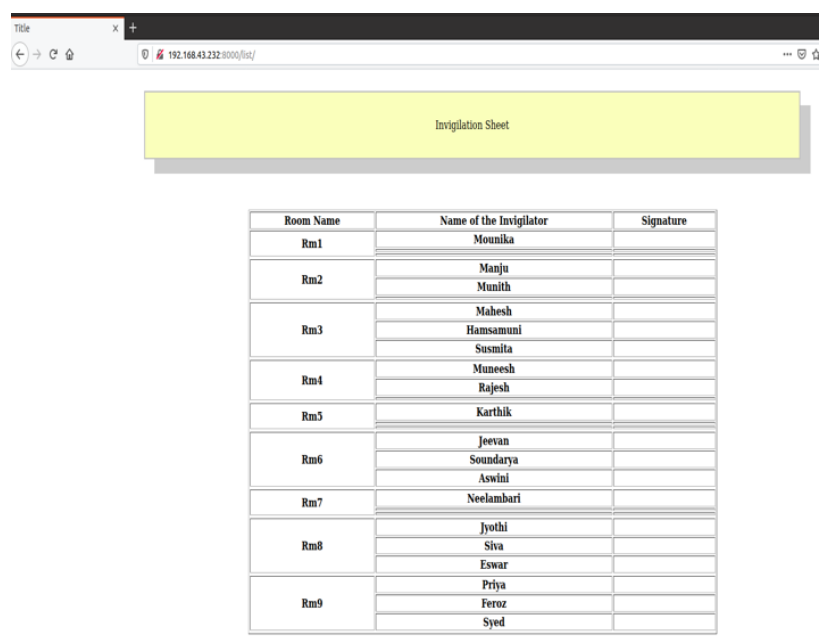

Fig 34. Invigilator Allotment

\section{Conclusion}

All the academic seminaries are appraising the mastery of students through tests or Examinations. Devising the students and invigilators into the examination halls are the part of proper conduction of Examinations. The number of invigilators in the examination hall depends on the number of students. The number of students depends on the seating capacity of the room. Available rooms are presented for a few section students having different examinations. The algorithm automatically picks the rooms and students and successfully allot so that no student of the same section are side by side or back to back or diagonal in all cases except for the last room. The camera placed inside the rooms take the pictures and sent to the server continuously. The Django server identifies the images of the specific room. The next step is to process the images for validation of students. The best face detector is needed for finding the faces. The face detector must have the capability to find the faces at speed and have very good accuracy. The three best-performed face detectors are chosen, which are Haar Cascade, HoG and $\mathrm{CNN}$. It is found from speed analysis that $\mathrm{CNN}$ is too slow to use with CPUs. In terms of speed, Haar method is best, but it gives more false positives. Hence the HoG method is chosen for face detection. For recognising the faces, the faces from face detector are matched with stored faces in the database. The face recognizer matches the face by finding the Euclidean distance of their 128D embeddings and comparing with the threshold. The face recognizer can recognize the faces with $99.38 \%$ accuracy. Hence, achieved great success in predicting the right student for the right room.

This is an Open Access article distributed under the terms of the Creative Commons Attribution License.

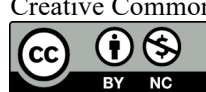

\section{References}

1. Jamal Hussain Shah, Muhammad Sharif, Mudassar Raza, Marryam Murtaza and Saeed-Ur-Rehman,2015,Robust Face Recognition Technique under Varying Illumination, Journal of Applied Research and Technology, https://doi.org/10.1016/S1665-6423(15)30008-0

2.Stan Z. Li, RuFeng Chu, ShengCai Liao, and Lun Zhang,2007,Illumination Invariant Face Recognition Using Near-
Infrared Images, IEEE Transactions on pattern analysis and machine intelligence, https://doi.org/10.1109/TPAMI.2007.1014.

3. iwen Lu, Venice Erin Liong,Xiuzhuang Zhou, and Jie Zhou, 2015,Learning Compact Binary Face Descriptor for Face Recognition, IEEE Transactions on Pattern Analysis and Machine Intelligence, https://doi.org/10.1109/TPAMI.2015.2408359. 
4. Shwetank Arya, Neeraj Pratap, Karamjit Bhatia,2015, Future of Face Recognition: A Review, Second International Symposium on Computer Vision and the Internet(VisionNet'15), https://doi.org/10.1016/j.procs.2015.08.076.

5. Unsang Park, Yiying Tong, and Anil K. Jain,2010,Age-Invariant Face Recognition, IEEE Transactions on Pattern Analysis and Machine Intelligence, https://doi.org/10.1109/TPAMI.2010.14.

6.Changxing Ding, Chang Xu, and Dacheng Tao, 2015, Multi-task PoseInvariant Face Recognition, IEEE Transactions on Image processing, https://doi.org/10.1109/TIP.2015.2390959.

7.Zehra Kamisli ozturk, Gurkan Ozturk, Mujgan Sagir, 2019, An Automated multi-objective invigilator-exam assignment system, International Journal of Information Technology \& Decision Making, https://doi.org/10.1142/S0219622010003798

8. Noeman Mirza,and Eric Staples,February 2010, Webcam as a New Invigilation Method: Students' Comfort and Potential for Cheating, Journal of Nursing Education, https://doi.org/10.3928/01484834-20090916-06. .

9. Farida Hanum, Mira A. Romliyah, Toni Bakhtiar2015,Exam Invigilators Assignment Problem: A Goal Programming Approach,Applied Mathematical Sciences, https://doi.org/10.12988/AMS.2015.52134.

10. M Paresa Beham. S Mahammod Mansur 2013, A Review of face recognition methods, International Journal of Pattern Recognition and Artificial https://doi.org/10.1142/S0218001413560053.

11.Paulo Menezes, Jos'eCarlosBarreto 2004,Face tracking based on HAAR-like features and eigenfaces, 5th IFAC/EURON Symposium on Intelligent Autonomous Vehicles Instituto Superior Técnico, Lisboa, Portugal, https://oi.org/10.1016/S1474-6670(17)31993-6

12. A. Alvarado, B. Bajaña, J. A. Munoz and W. Velásquez, 2017, Bicycle Protection within an University Area using Geolocation and Perimeter Security, IEEE Latin America Transactions,https://doi.org/10.1109/TLA.2017.7932702.

13.Andrea Sanna, Fabrizio Lamberti,Gianluca Paravati, and Claudio Demartini,2012, Automatic Assessment of 3D Modeling Exams, IEEE Transactions on learning technologies, https://doi.org/10.1109/TLT.2011.4.

14.Im Y. Jung and Heon Y. Yeom,AUGUST 2009,Enhanced Security for Online Exams Using Group Cryptography, IEEE Transactions on education, https://doi.org/10.1109/TE.2008.928909.

15.Mustafa Kaiiali, Armagan Ozkaya, Halis Altun, Hatem Haddad, and Marc Alier, 2016, Designing a Secure Exam Management System (SEMS) for M-Learning Environments, IEEE Transactions on learning technologies, https://doi.org/10.1109/TLT.2016.2524570.
16.Gabriel Abriel Hermosilla Vigneau, Jose Luis Verdugo, Gonzalo farias castro, Francisco Pizarro, Esteban Vera, 2017, Thermal Face Recognition Under Temporal Variation Conditions, IEEE Translations and content mining, https://doi.org/10.1109/ACCESS.2017.2704296.

17. Yousef Atoum, Liping Chen, Alex X. Liu, Stephen D. H. Hsu, and Xiaoming Liu,JULY 2017,Automated Online Exam Proctoring, IEEE Transactions on multimedia, https://doi.org/10.1109/TMM.2017.2656064.

18. Yun-Fu Liu, Member, Jing-Ming Guo, Po-Hsien Liu, Jiann-Der Lee, and Chen-Chieh Yao, 2018, Panoramic Face Recognition, IEEE Trnsactions on circuits ans systems for video technology, https://doi.org/10.1109/TCSVT.2017.2693682.

19.Issam Dagher and Rabih Nachar, JUNE 2006,Face Recognition Using IPCA-ICA Algorithm, IEEE Transactions on pattern analysis and machine intelligence, https://doi.org/10.1109/TPAMI.2006.118.

20.R. Raghavendra, Kiran B. Raja, and Christoph Busch, 2015, Presentation Attack Detection for Face Recognition Using Light Field Camera, IEEE Transactions on image processing, https://doi.org/10.1109/TIP.2015.2395951

21. Xiaoli Zhou and Bir Bhanu, 2007, Integrating Face and Gait for Human Recognition at a Distance in Video, IEEE Transactions on systems, man and cybernetics, https://doi.org/10.1109/TSMCB.2006.889612.

22. Jing Huang, Yunyi Shang and Hai Chen, 2019, Improved ViolaJones face detection algorithm based on HoloLens, EURASIP Journal on Image and Video Processing, https://doi.org/10.1186/s13640-019-0435-6.

23. Wenqi Wu, Yingjie Yin, Xingang Wang, 2019. Face Detection With Different Scales Based on Faster R-CNN, IEEE Transactions on Cybernetics, https://doi.org/10.1109/TCYB.2018.2859482.

24. Nandini, V., Uma Maheswari, P. 2020, Automatic assessment of descriptive answers in online examination system using semantic relational features. J Supercomput 76, 4430-4448 https://doi.org/10.1007/s11227-018-2381-y

25. Rahman, S., Rahman, M.M., Abdullah-Al-Wadud, M. et al. An adaptive gamma correction for image enhancement. J Image Video Proc. 2016, 35 (2016). https://doi.org/10.1186/s13640-016-0138-1

26. SivaSai J.G., Srinivasu P.N., Sindhuri M.N., Rohitha K., Deepika S. 2021, An Automated Segmentation of Brain MR Image Through Fuzzy Recurrent Neural Network. In: Bhoi A., Mallick P., Liu CM., Balas V. (eds) Bio-inspired Neurocomputing. Studies in Computational Intelligence, vol 903. Springer, Singapore. https://doi.org/10.1007/978-981-15-5495-7 9 